\title{
Electric field dependent radiative decay kinetics of polar InGaN/GaN quantum heterostructures at low fields
}

Emre Sari, Sedat Nizamoglu, In-Hwan Lee, Jong-Hyeob Baek, and Hilmi Volkan Demir

Citation: Appl. Phys. Lett. 94, 211107 (2009);

View online: https://doi.org/10.1063/1.3142386

View Table of Contents: http://aip.scitation.org/toc/apl/94/21

Published by the American Institute of Physics

\section{Articles you may be interested in}

Temperature-dependent emission intensity and energy shift in InGaN/GaN multiple-quantum-well lightemitting diodes

Applied Physics Letters 82, 3614 (2003); 10.1063/1.1578539

Polarization effects in photoluminescence of $C$ - and $M$-plane GaN/AIGaN multiple quantum wells Applied Physics Letters 81, 4130 (2002); 10.1063/1.1524298

Photoluminescence imaging of silicon wafers

Applied Physics Letters 89, 044107 (2006); 10.1063/1.2234747

Efficient nonradiative energy transfer from InGaN/GaN nanopillars to CdSe/ZnS core/shell nanocrystals Applied Physics Letters 98, 163108 (2011); 10.1063/1.3562035

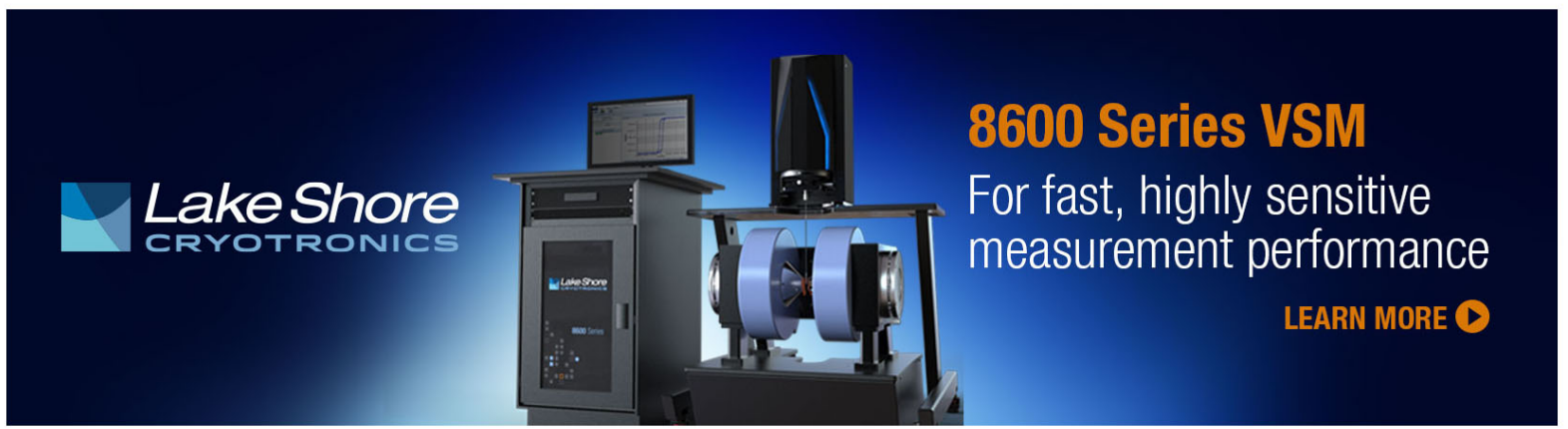




\title{
Electric field dependent radiative decay kinetics of polar InGaN/GaN quantum heterostructures at low fields
}

\author{
Emre Sari, ${ }^{1,2,3}$ Sedat Nizamoglu, ${ }^{1,2,3}$ In-Hwan Lee, ${ }^{4}$ Jong-Hyeob Baek, ${ }^{5}$ and \\ Hilmi Volkan Demir ${ }^{1,2,3,6, a)}$ \\ ${ }^{1}$ Department of Electrical and Electronics Engineering, Bilkent University, Ankara, \\ TR-06800 Bilkent, Turkey \\ ${ }^{2}$ Nanotechnology Research Center, Bilkent University, Ankara, TR-06800 Bilkent, Turkey \\ ${ }^{3}$ Institute of Materials Science and Nanotechnology, Bilkent University, Ankara, TR-06800 Bilkent, Turkey \\ ${ }^{4}$ School of Advanced Materials Engineering, Research Center of Industrial Technology, \\ Chonbuk National University, Chonju 561-756, Republic of Korea \\ ${ }^{5}$ Korea Photonics Technology Institute, Gwangju 500-460, Republic of Korea \\ ${ }^{6}$ Department of Physics, Bilkent University, Ankara, TR-06800 Bilkent, Turkey
}

(Received 9 March 2009; accepted 3 May 2009; published online 29 May 2009)

\begin{abstract}
Electric field dependent photoluminescence decay kinetics and its radiative component are studied in polar InGaN/GaN quantum heterostructures at low fields. Under externally applied electric field lower than polarization fields, spectrally and time resolved photoluminescence measurements are taken to retrieve internal quantum efficiencies and carrier lifetimes as a function of the applied field. Subsequently, relative behavior of radiative recombination lifetimes is obtained in response to the applied field. In these characterizations of polar InGaN/GaN structures, we observe that both the carrier lifetime and the radiative recombination lifetime decrease with increasing external electric field, with the radiative component exhibiting weaker field dependence. (C) 2009 American Institute of Physics. [DOI: 10.1063/1.3142386]
\end{abstract}

In the past decade, optoelectronic devices based on IIInitride quantum structures including high-brightness light emitting diodes, ${ }^{1-3}$ laser diodes, ${ }^{4-6}$ and electroabsorption modulators $^{7-9}$ have been demonstrated. These devices have drawn strong attention thanks to their important applications in solid-state lighting, high density data storage, printing, packaging, and nonline-of-sight communications. InGaN/ GaN quantum structures have also been widely studied in the same time span to develop a better understanding of the underlying physics and improve their device operation in the visible and near ultraviolet. ${ }^{10,11}$ For optoelectronic devices, one important physical feature is the radiative recombination. Although the carrier dynamics have been discussed previously as a function of externally applied electric field in Ref. 12, the electric field dependent radiative recombination in particular has not been studied systematically to date.

Here, in this letter, we present our systematic investigation on determining relative changes in radiative recombination lifetimes of polar InGaN/GaN quantum heterostructures in response to the applied electric field. In our study, the external electric field levels that we applied across the quantum structures is in opposite direction to and one order of magnitude less than the calculated polarization-induced built-in electric field inside the quantum well layers $\left(E_{\text {well }}\right.$ $\approx 190 \mathrm{~V} / \mu \mathrm{m}$ ). The maximum applied electric field is limited by the photoluminescence (PL) optical output power levels that are detectable in our experimental setup. On the other hand, the applied field is in the same direction and of the same order of magnitude as the polarization field in the barrier layers $\left(E_{\text {barrier }} \approx 70 \mathrm{~V} / \mu \mathrm{m}\right)$. At these external field levels, our experimental results reveal a decrease in the in-

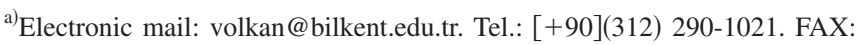
[+90](312) 290-1015.
}

ternal quantum efficiencies (QEs), carrier lifetimes, and radiative recombination lifetimes with increasing field, as verified consistently with steady state and transient PL measurements of these polar quantum structures.

For our investigation, we grew an $\mathrm{InGaN} / \mathrm{GaN}$ quantum heterostructure embedded in a $p-i-n$ diode architecture on $c$-plane sapphire substrate by using metal-organic chemical vapor deposition. The epitaxial design was developed for a light emitting diode operating at $448 \mathrm{~nm}$. Its active layers included five periods of $2.5 \mathrm{~nm}$ thick $\mathrm{In}_{0.15} \mathrm{Ga}_{0.85} \mathrm{~N}$ quantum well and $7.5 \mathrm{~nm}$ thick GaN barrier layers grown in the intrinsic region of the epitaxy. Surrounding the intrinsic layer are the $n$-type $(\mathrm{Si}: \mathrm{GaN})$ and $p$-type $(\mathrm{Mg}: \mathrm{GaN})$ layers grown with their targeted doping concentrations of $5 \times 10^{18}$ and 8 $\times 10^{17} \mathrm{~cm}^{-3}$ and thicknesses of $\sim 2$ and $\sim 0.2 \mu \mathrm{m}$, respectively. This design allows for partially compensating for the polarization-induced electrostatic field inside the well layers in reverse bias operation. For the application of external electric field, standard mesa and electrode fabrication cycles were implemented. Reactive ion etching and metallization steps were followed by dicing and transistor outline (TO) packaging of $300 \times 300 \mu \mathrm{m}^{2}$ devices. For Ohmic contact formation to $n$-type (bottom) layer 10/200 nm Ti/Al was used, whereas for $p$-type (top) layer, a $100 \mathrm{~nm}$ thick indium tin oxide (ITO) was used to obtain optically semitransparent windows. These ITO based semitransparent contacts enabled a uniform application of the electric field across the active layers while maintaining a low optical loss for the incident light due to its low background absorption. On top of ITO, a 10/100 nm Ti/Au layers were further used to make strong $\mathrm{Au}$ wire bonds to the TO can.

We performed time resolved PL (TRPL) measurements (using PicoQuant PicoHarp 300) at room temperature to determine the electrical dependence of decay kinetics. The optical apparatus consists of a commercially available InGaN/ 


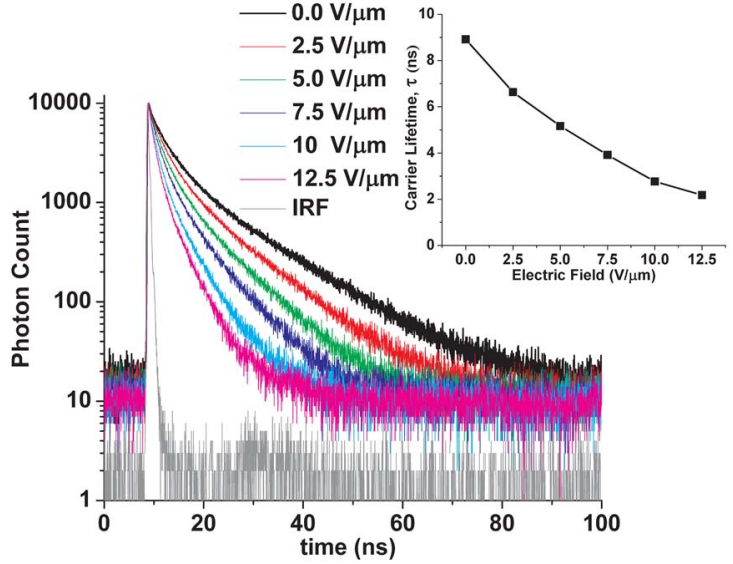

FIG. 1. (Color online) TRPL spectra of our polar InGaN/GaN quantum heterostructure under different electric field levels. The inset shows the carrier lifetime vs. applied electric field as a result of the TRPL analysis.

GaN based near-ultraviolet $(375 \mathrm{~nm})$ laser diode used in pulsed mode operation, a monochromator, a photomultiplier tube, a high speed photodetector, and controller electronics. Figure 1 shows the TRPL spectra of our polar InGaN/GaN quantum structure under different levels of externally applied electric field. Here we are interested in the radiative decay of photons whose energies correspond to the transition energy between the electron and hole ground states. This photon energy is $2.76 \mathrm{eV}$, corresponding to $448 \mathrm{~nm}$, the peak electroluminescence wavelength.

These experimental PL decays are numerically further analyzed by deconvolving the input pulse (instrumental response function) and using three best-fitting exponential decays in the fluorescence decay analysis software of PicoQuant (FLUOFIT). This numerical curve fitting procedure leads to the retrieval of intensity-averaged lifetime for our detailed understanding of carrier lifetime $(\tau)$ behavior, with a chi-square $\left(\chi^{2}\right)$ error $1.0 \pm 0.05$ for each PL decay in a particular field level. In these analyses, the levels of $\chi^{2}$ error were sufficiently close to unity to achieve accurate enough representation using triple-exponential decays, each with a single lifetime. In the inset of Fig. 1, we present the change of carrier lifetime under $0-1.25 \mathrm{~V}$ reverse bias voltages, corresponding to $0-12.5 \mathrm{~V} / \mu \mathrm{m}$ electric field swings. The strong electric field dependent decrease of carrier lifetime is in agreement with the previous work of Jho et al. ${ }^{12}$ on carrier dynamics. The rate of change of carrier lifetime monotonically decreases with increasing field from $0.91 \mathrm{~ns} / \mathrm{V} \mu \mathrm{m}$ (for $0-2.5 \mathrm{~V} / \mu \mathrm{m}$ field swings) to $0.23 \mathrm{~ns} / \mathrm{V} \mu \mathrm{m}$ (for $10-12.5 \mathrm{~V} / \mu \mathrm{m}$ field swings). We believe such strong dependence of carrier lifetime on the external electric field originates from its nonradiative component for the most part, due to relatively low absolute $\mathrm{QE}(\mathrm{QE}$ $<10 \%)$.

The radiative component $\left(\tau_{r}\right)$ of the overall PL decay is related to the QE as given in Eq. (1). In addition to the carrier lifetime measurements, to determine the electric field dependence of $\tau_{r}$, we performed steady state PL measurements. This PL characterization was carried out on the same device, under the same external electric field levels, using a cw $\mathrm{He}-\mathrm{Cd}$ laser as the excitation source operating at a wavelength of $325 \mathrm{~nm}$ and collected via a UV-visible photodetector placed after a high resolution monochromator. Here,

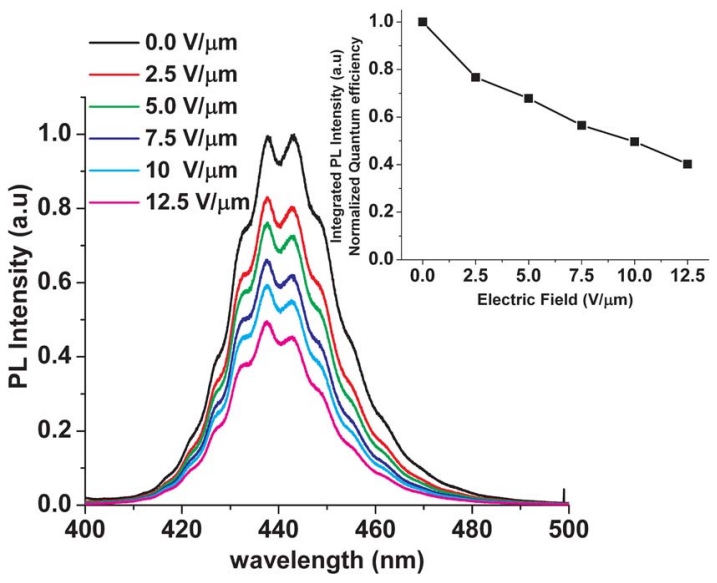

FIG. 2. (Color online) Steady-state PL spectra of our polar InGaN/GaN quantum heterostructure under different electric field levels. The integrated PL intensity for the corresponding electric field level is presented in the inset.

since we consider only relative QEs, it is important to make sure that the experimental conditions are identical for different external electric field levels. Also, as apparent from the constant fringe periods in the PL spectra, the refractive index change due to electro-optic effect is insignificant at the applied electric field levels, making it possible to ignore the effect of extraction efficiency on relative QEs in our analyses. Also, since the external electric field builds up across the intrinsic $(i)$ region of our epitaxial structure, it is only the $i$-region where optical processes are affected by the applied electric field in our experiments. Hence, optical absorption taking place in other layers is assumed to be unaffected from the external field change in our analyses,

$$
\mathrm{QE}(E)=\frac{N_{e}(E)}{N_{a}} \propto \frac{\tau(E)}{\tau_{r}(E)} .
$$

The electric field dependent steady state PL measurement results are given in Fig. 2. The PL spectra exhibit a narrowing particularly with the longer wavelength part of the spectra shifting with the applied field due to partial compensation of polarization-induced built-in electric field inside the well layers. The normalized PL spectra depicted in Fig. 3 clearly show this blueshift at the longer wavelengths, as a result of the reversed quantum confined Stark effect. ${ }^{8,13,14}$ This is an

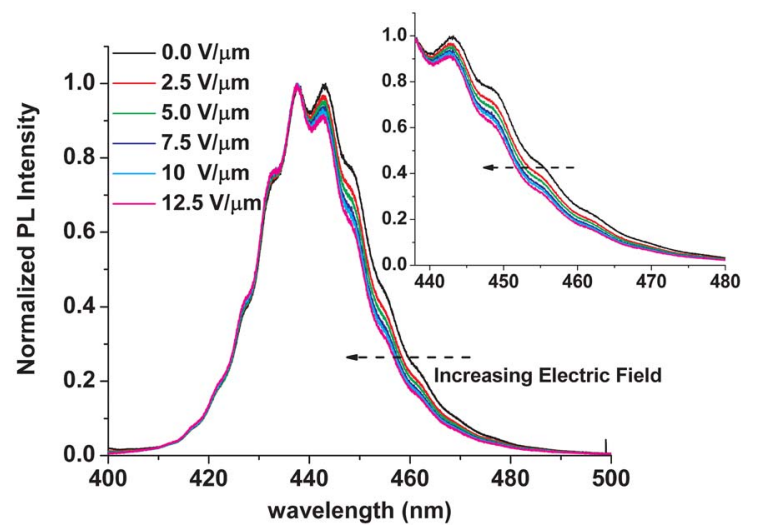

FIG. 3. (Color online) Normalized PL spectra of our polar InGaN/GaN quantum heterostructure under electric field. We observe a narrowing of the spectra along with a blueshift in the longer wavelength region, with a zoom-in presented in the inset for clarity. 


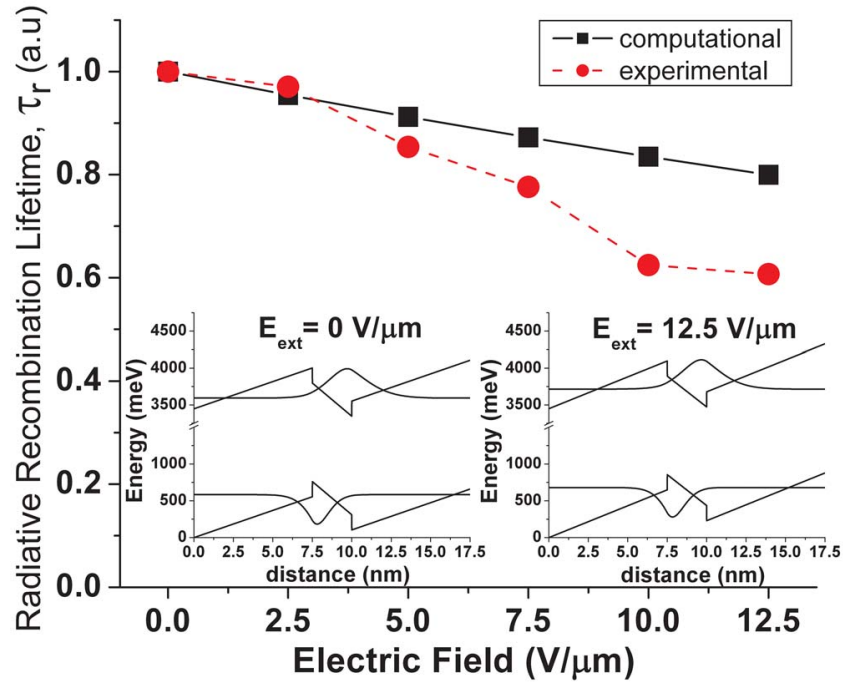

FIG. 4. (Color online) Relative radiative recombination lifetime extracted from TRPL and PL measurements. The inset shows energy band diagrams corresponding electron and hole ground state wave functions for the case of $0 \mathrm{~V} / \mu \mathrm{m}$ and $12.5 \mathrm{~V} / \mu \mathrm{m}$ externally applied electric field.

apparent sign that electric field dependence of transition energy between the ground state electron and hole wave functions $(\langle e 1|H| h 1\rangle)$ is stronger than those between higher order states. From the definition of QE given in Eq. (1), we understand that the QE decreases with decreasing number of emitted photons $\left(N_{e}\right)$ in response to increasing external electric field, assuming that the application of electric field does not change the number of absorbed photons $\left(N_{a}\right)$. This condition is valid at such short excitation wavelengths in the ultraviolet for the low external electric field levels that we use in our characterization. The inset of Fig. 2 shows the electric field dependence of PL intensity integrated over 440-470 nm corresponding to $2.73 \pm 0.09 \mathrm{eV}$. This energy range we considered in this partial integration spans the transition energy of ground state electron and hole for all electric field values. This shows the relative QE behavior as a function of applied field.

From the relative QE and carrier lifetime measurements, we can deduce relative radiative recombination lifetimes. As shown in Fig. $4, \tau_{r}$ tends to decrease with increasing external electric field. The decrease of radiative recombination lifetime is explained with the overlap integral of electron and hole ground states (calculated using transfer matrix method) that increases with increasing external electric field as given for two of our cases in the inset of Fig. 4. This is a result of the reversed quantum confined Stark effect and Fermi's golden rule. Also in Fig. 4, we compare the normalized reciprocal of squared overlap integral of calculated electron and hole ground state wave functions with our experimental relative $\tau_{r}$ results, both of which show the same trend of change in response to the applied field.

In conclusion, we presented radiative decay kinetics of polar InGaN/GaN quantum heterostructures and its electric field dependence at low applied fields. Our experimental measurements and numerical analyses show that the carrier lifetimes and radiative recombination lifetimes both decrease with increasing external electric field. However, the radiative component demonstrates comparatively a weaker dependence on the electric field, while the overall PL kinetics exhibits a stronger electric field dependence. As a result of our study, we qualitatively demonstrate and verify a fundamental physics concept, Fermi's golden rule, to be dependent on external electric field in a polar InGaN/GaN quantum heterostructure.

${ }^{1}$ S. Nakamura, M. Senoh, N. Iwasa, and S. Nagahama, Appl. Phys. Lett. 67, 1868 (1995).

${ }^{2}$ C. Huh, H.-S. Kim, S.-W. Kim, J.-M. Lee, D.-J. Kim, I.-H. Lee, and S.-J. Park, J. Appl. Phys. 87, 4464 (2000).

${ }^{3}$ E. F. Schubert and J. K. Kim, Science 308, 1274 (2005).

${ }^{4}$ S. Nakamura, M. Senoh, S. Nagahama, N. Iwasa, T. Yamada, T. Matsushita, Y. Sugimoto, and H. Kiyoku, Appl. Phys. Lett. 69, 4056 (1996).

${ }^{5}$ M. Hansen, P. Fini, L. Zhao, A. C. Abare, L. A. Coldren, and J. S. Speck, Appl. Phys. Lett. 76, 529 (2000).

${ }^{6}$ S. Nakamura, S. J. Pearton, and G. Fasol, The Blue Laser Diode (Springer, Berlin, 1997).

${ }^{7}$ M. Kneissl, T. L. Paoli, P. Kiesel, D. W. Treat, M. Teepe, N. Miyashita, and N. M. Johnson, Appl. Phys. Lett. 80, 3283 (2002).

${ }^{8}$ E. Sari, T. Ozel, S. Nizamoglu, and H. V. Demir, Appl. Phys. Lett. 90, 011101 (2007).

${ }^{9}$ T. Ozel, E. Sari, S. Nizamoglu, and H. V. Demir, J. Appl. Phys. 102, 113101 (2007).

${ }^{10}$ V. Fiorentini, F. Bernardini, F. D. Sala, A. Di Carlo, and P. Lugli, Phys. Rev. B 60, 8849 (1999).

${ }^{11}$ T. Takeuchi, C. Wetzel, S. Yamaguchi, H. Sakai, H. Amano, I. Akasaki, Y. Kaneko, S. Nakagawa, Y. Yamaoka, and N. Yamada, Appl. Phys. Lett. 73, 1691 (1998).

${ }^{12}$ Y. D. Jho, J. S. Yahng, E. Oh, and D. S. Kim, Phys. Rev. B 66, 035334 (2002).

${ }^{13}$ D. A. B. Miller, D. S. Chemla, T. C. Damen, A. C. Gossard, W. Wiegmann, T. H. Wood, and C. A. Burrus, Phys. Rev. B 32, 1043 (1985).

${ }^{14}$ L. I. Gurinovich, M. V. Artemyev, U. Woggon, Physics, Chemistry and Applications of Nanostructures (Reviews and Short Notes to Nanomeeting-2001), 2001 (unpublished), pp. 150-155. 